\title{
On one approach to the numerical modeling of the strain-stress state of layered rock mass
}

\author{
Alexander Baryakh \\ Perm Federal Research Center of Ural Branch of Russian Academy of Science, director, corresponding member of Russian Academy \\ of Science, Russia \\ bar@mi-perm.ru,bttp:/ / mww.permsc.rul
}

Sergey Lobanov

Mining Institute of the Ural Branch of the Russian Academy of Science, Russia

LSerg@mi-perm.ru, bttps://www.mi-perm.ru/

\begin{abstract}
According to the principle of virtual work for a system of elastic layers, a semi-analytical scheme for constructing the finite-dimensional analog of the variational equation was developed based on the well-known analytical solution in the Fourier series for a single layer. The solution of a test problem shows that the constructed numerical procedure of mathematical modeling ensures fairly accurate calculations and highly effective computer-aided realization. It was demonstrated that the proposed semi-analytical scheme for constructing the finite-dimensional analog of the variational equation can be effectively used for the assessment of safety conditions of the potash salt development within the Upper Kama salt deposit.
\end{abstract}

KEYWORDS. Mathematical modeling; Finite elements; Strains; Stresses; Rocks.

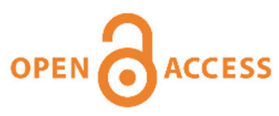

Citation: Baryakh, A., Lobanov., S., On one approach to the numerical modeling of the strain-stress state of layered rock mass, Frattura ed Integrità Strutturale, 49 (2019) 257-266.

Received: 29.04 .2019

Accepted: 21.05 .2019

Published: 01.07.2019

Copyright: (C) 2019 This is an open access article under the terms of the CC-BY 4.0, which permits unrestricted use, distribution, and reproduction in any medium, provided the original author and source are credited.

\section{INTRODUCTION}

$\mathrm{S}$ edimentary rock masses, the interior of which often contains salt deposits, are characterized by a thin-layered structure. The analysis of their strain-stress state and failure as a result of mining operations is of considerable importance in ensuring the protection of mines against flooding [1,2]. In certain cases such rock masses can be represented as a system of plane-parallel layers. In solving the problems of this class, it is common practice to use both the traditional numerical finite and boundary element methods and special computational techniques, which are based on the "sequential stiffness" and "sequential compliance" procedures [3], the Fourier series expansion [4], and combinations of various computational procedures [5.6]. These methods were thoroughly discussed and analyzed in work [7]. 


\section{FINITE-DIMENSIONAL ANALOG CONSTRUCTION METHOD}

onsider a system of $N$ plane-parallel elastic layers of arbitrary thickness $2 h_{n}$, which is under plane strain conditions (Fig. 1). There are no mass forces. Due to the fact that these forces are usually constant, they, if necessary, can be easily taken into account based on the usual superposition. Let us assume that homogeneous conditions, for example, zero displacements, are preset at the distant side boundaries. In addition, for the sake of simplicity we will assume that cohesion between the contact layers is perfect. The displacement boundary conditions are as follows: $\{U\}=\{\bar{U}\}$ for $S$. Here and in all following examples there is a line of symmetry at the left boundary.

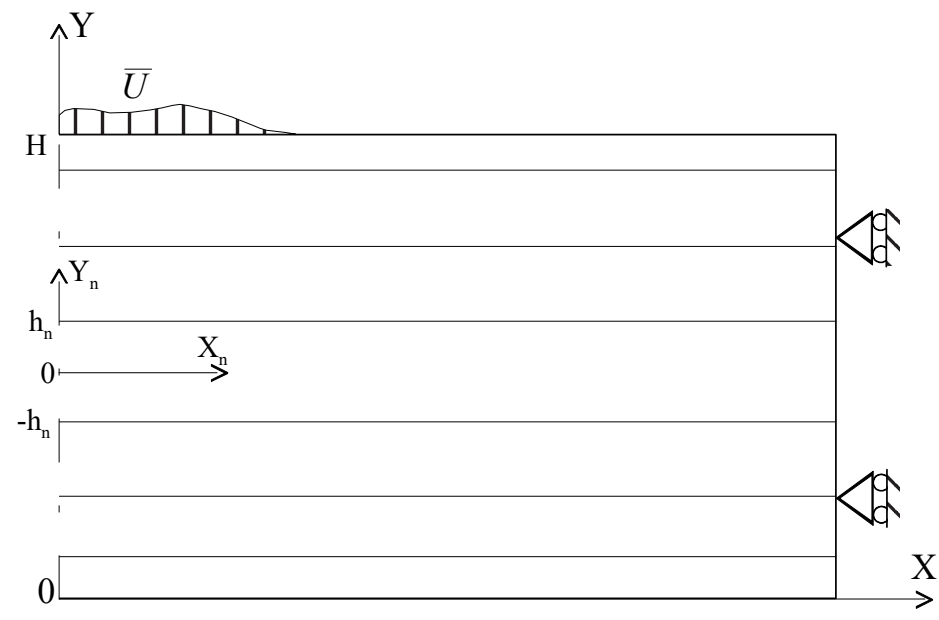

Figure 1: General computational scheme

Let us write down the principle of additional virtual work [8]:

$$
\int_{V}\{\delta \sigma\}^{T}\{\varepsilon\} d V=\int_{S}\{\delta T\}^{T}\{\bar{U}\} d S
$$

where $\{\varepsilon\},\{\sigma\}$ - strain and stress vectors $\{\delta T\}=\left\{\delta \sigma_{i j}\right\}^{T}\{n\}$ respectively, $\mathrm{n}$ - cosine of the outer normal to $S$. As is known [8], this variational principle is true for arbitrary infinitely small stress variations that satisfy the equilibrium equations and the specified stress boundary conditions.

For each layer in the local Cartesian coordinate system $x 0 y$ (the $x$ axis runs across the middle line of a layer) stress at any point can be represented as a Fourier series expansion:

$$
\{\sigma(x, y)\}=\sum_{k=1}^{\infty}\left[t_{k}^{c}(x)\right]\left\{\sigma_{k}^{c}(y)\right\}+\sum_{k=1}^{\infty}\left[t_{k}^{a}(x)\right]\left\{\sigma_{k}^{a}(y)\right\}
$$

where

$$
\begin{aligned}
& \sigma(x, y)=\left[\begin{array}{l}
\sigma_{x x}(x, y) \\
\sigma_{y y}(x, y) \\
\tau_{x y}(x, y)
\end{array}\right], \quad \sigma_{k}^{c(a)}=\left[\begin{array}{l}
\sigma_{x x k}^{c(a)}(y) \\
\sigma_{y y k}^{c(a)}(y) \\
\tau_{x y k}^{c(a)}(y)
\end{array}\right] \\
& {\left[t_{k}^{c}(x)\right]=\left[\begin{array}{ccc}
\cos \alpha_{k} x & 0 & 0 \\
0 & \cos \alpha_{k} x & 0 \\
0 & 0 & \sin \alpha_{k} x
\end{array}\right],\left[t_{k}^{a}(x)\right]=\left[\begin{array}{ccc}
\sin \alpha_{k} x & 0 & 0 \\
0 & \sin \alpha_{k} x & 0 \\
0 & 0 & \cos \alpha_{k} x
\end{array}\right]}
\end{aligned}
$$


$\alpha_{k}=\pi k / l ; 2 l$ - interval of expansion into the Fourier series, upper indexes $c$ and $a$ denote, respectively, the values related to the symmetric and antisymmetric state of a layer in relation to the $y$ axis.

The Fourier coefficients in expansion (2) can be expressed as constants within the layer of vectors $\left\{C_{k}^{c}\right\}$ and $\left\{C_{k}^{a}\right\}[9]$ :

$$
\left\{\sigma_{k}^{c}(y)\right\}=\left[r_{k}(y)\right]\left\{C_{k}^{c}\right\} ;\left\{\sigma_{k}^{a}(y)\right\}=\left[r_{k}(y)\right]\left\{C_{k}^{a}\right\}
$$

where $\left[r_{k}(y)\right]$ - the known matrix [6],

$$
\left[r_{k}(y)\right]=\alpha_{k}\left[\begin{array}{cccc}
\alpha_{k} \cdot \operatorname{sh}\left(\alpha_{k} y\right) & \alpha_{k} \cdot \operatorname{ch}\left(\alpha_{k} y\right) & \alpha_{k} y \cdot \operatorname{sh}\left(\alpha_{k} y\right)+2 \cdot \operatorname{ch}\left(\alpha_{k} y\right) & \alpha_{k} y \cdot \operatorname{ch}\left(\alpha_{k} y\right)+2 \cdot \operatorname{sh}\left(\alpha_{k} y\right) \\
-\alpha_{k} \cdot \operatorname{sh}\left(\alpha_{k} y\right) & -\alpha_{k} \cdot \operatorname{ch}\left(\alpha_{k} y\right) & -\alpha_{k} y \cdot \operatorname{sh}\left(\alpha_{k} y\right) & -\alpha_{k} y \cdot \operatorname{ch}\left(\alpha_{k} y\right) \\
\alpha_{k} \cdot \operatorname{ch}\left(\alpha_{k} y\right) & \alpha_{k} \cdot \operatorname{sh}\left(\alpha_{k} y\right) & \alpha_{k} y \cdot \operatorname{ch}\left(\alpha_{k} y\right)+\operatorname{sh}\left(\alpha_{k} y\right) & \alpha_{k} y \cdot \operatorname{sh}\left(\alpha_{k} y\right)+\operatorname{ch}\left(\alpha_{k} y\right)
\end{array}\right]
$$

The representation of stresses in the form of expansion (2), with (3) taken into account, satisfies the equilibrium equations, which, as already noted, is an integral condition for the validity of the principle of virtual work.

Let us express constants $C_{k}^{c(a)}$ in (3) in terms of the relevant coefficients of decomposition of force vectors $p_{k}^{c(a)}$ at the upper $p_{k t}^{c(a)}$ and lower $p_{k b}^{c(a)}$ boundaries of a layer:

$$
\left\{C_{k}^{c(a)}\right\}=\left[R_{k 0}\right]^{-1}\left\{p_{k}^{c(a)}\right\}
$$

where $\left\{p_{k}^{c(a)}\right\}=\left\{p_{k t}^{c(a)}, p_{k b}^{c(a)}\right\}$;

$$
\begin{aligned}
& \left\{p_{k t}^{c(a)}\right\}=\left\{\begin{array}{c}
\sigma_{y y k}^{c(a)}(b) \\
\tau_{x y k}^{c(a)}(b)
\end{array}\right\} ;\left\{p_{k b}^{c(a)}\right\}=\left\{\begin{array}{c}
\sigma_{y y k}^{c(a)}(-b) \\
\tau_{x y k}^{c(a)}(-b)
\end{array}\right\} \\
& {\left[\mathrm{R}_{k 0}\right]=\left[\begin{array}{c}
r_{k}^{*}(b) \\
r_{k}^{*}(-b)
\end{array}\right]} \\
& {\left[r_{k}^{*}(y)\right]=\alpha_{k}\left[\begin{array}{cccc}
-\alpha_{k} \cdot \operatorname{sh}\left(\alpha_{k} y\right) & -\alpha_{k} \cdot \operatorname{ch}\left(\alpha_{k} y\right) & -\alpha_{k} y \cdot \operatorname{sh}\left(\alpha_{k} y\right) & -\alpha_{k} y \cdot \operatorname{ch}\left(\alpha_{k} y\right) \\
\alpha_{k} \cdot \operatorname{ch}\left(\alpha_{k} y\right) & \alpha_{k} \cdot \operatorname{sh}\left(\alpha_{k} y\right) & \alpha_{k} y \cdot \operatorname{ch}\left(\alpha_{k} y\right)+\operatorname{sh}\left(\alpha_{k} y\right) & \alpha_{k} y \cdot \operatorname{sh}\left(\alpha_{k} y\right)+\operatorname{ch}\left(\alpha_{k} y\right)
\end{array}\right] .}
\end{aligned}
$$

Then, the expression for stresses (2) can be represented as:

$$
\{\sigma(x, y)\}=\sum_{k=1}^{\infty}\left[t_{k}^{c}(x)\right]\left[r_{k}(y)\right]\left[R_{k 0}\right]^{-1}\left\{p_{k}^{c}\right\}+\sum_{k=1}^{\infty}\left[t_{k}^{a}(x)\right]\left[r_{k}(y)\right]\left[R_{k 0}\right]^{-1}\left\{p_{k}^{a}\right\}
$$

and the strains will be determined by the following relation:

$$
\{\varepsilon(x, y)\}=\sum_{k=1}^{\infty}[C]\left[t_{k}^{c}(x)\right]\left[r_{k}(y)\right]\left[R_{k 0}\right]^{-1}\left\{p_{k}^{c}\right\}+\sum_{k=1}^{\infty}[C]\left[t_{k}^{a}(x)\right]\left[r_{k}(y)\right]\left[R_{k 0}\right]^{-1}\left\{p_{k}^{a}\right\}
$$

where $[C]$ - compliance matrix that is for an isotropic layer with Young's modulus E and Poisson's ratio $v$ is written as: 


$$
[C]=\frac{1+v}{E}\left[\begin{array}{ccc}
1-v & -v & 0 \\
-v & 1-v & 0 \\
0 & 0 & 2
\end{array}\right]
$$

Based on the constructed relations for stresses (5) and strains (6), the left part of Eqn. (1) determining the virtual work of strains can be represented as follows:

$$
\begin{aligned}
& \int_{V}\{\delta \sigma(x, y)\}^{T}\left\{\varepsilon(x, y\} d V=\sum_{k=1}^{\infty} \sum_{m=1}^{\infty}\left(\left\{\delta p_{k}^{c}\right\}^{T}\left(\left[R_{k 0}\right]^{-1}\right)^{T} \int_{V}\left[r_{k}(y)\right]^{T}\left[t_{k}^{c}(x)\right]^{T}[C]\left[t_{m}^{c}(x)\right]\left[r_{m}(y)\right] d V\left[R_{m 0}\right]^{-1}\left\{p_{m}^{c}\right\}+\right.\right. \\
& +\left\{\delta p_{k}^{c}\right\}^{T}\left(\left[R_{k 0}\right]^{-1}\right)^{T} \int_{V}\left[r_{k}(y)\right]^{T}\left[t_{k}^{c}(x)\right]^{T}[C]\left[t_{m}^{a}(x)\right]\left[r_{m}(y)\right] d V\left[R_{m 0}\right]^{-1}\left\{p_{m}^{a}\right\}+ \\
& +\left\{\delta p_{k}^{a}\right\}^{T}\left(\left[R_{k 0}\right]^{-1}\right)^{T} \int_{V}\left[r_{k}(y)\right]^{T}\left[t_{k}^{a}(x)\right]^{T}[C]\left[t_{m}^{a}(x)\right]\left[r_{m}(y)\right] d V\left[R_{m 0}\right]^{-1}\left\{p_{m}^{a}\right\}+ \\
& \left.+\left\{\delta p_{k}^{a}\right\}^{T}\left(\left[R_{k 0}\right]^{-1}\right)^{T} \int_{V}\left[r_{k}(y)\right]^{T}\left[t_{k}^{a}(x)\right]^{T}[C]\left[t_{m}^{c}(x)\right]\left[r_{m}(y)\right] d V\left[R_{m 0}\right]^{-1}\left\{p_{m}^{c}\right\}\right)
\end{aligned}
$$

If in Eqn. (1):

$$
\{\delta T\}=\sum_{k=1}^{\infty}\left[t_{k}^{*_{c}}(x)\right]\left\{\delta \sigma_{k}^{c}\right\}+\sum_{k=1}^{\infty}\left[t_{k}^{*_{a}}(x)\right]\left\{\delta \sigma_{k}^{a}\right\}
$$

where

$$
\left[t_{k}^{*_{c}}\right]=\left[\begin{array}{cccc}
\cos \alpha_{k} x & 0 & 0 & 0 \\
0 & \sin \alpha_{k} x & 0 & 0 \\
0 & 0 & \cos \alpha_{k} \chi & 0 \\
0 & 0 & 0 & \sin \alpha_{k} x
\end{array}\right],\left[t_{k}^{*_{a}}\right]=\left[\begin{array}{cccc}
\sin \alpha_{k} x & 0 & 0 & 0 \\
0 & \cos \alpha_{k} x & 0 & 0 \\
0 & 0 & \sin \alpha_{k} x & 0 \\
0 & 0 & 0 & \cos \alpha_{k} x
\end{array}\right]
$$

then its right-hand part that determines the external virtual work is given as:

$$
\int_{S}\{\delta T\}^{T}\{\bar{U}\} d S=\sum_{k=1}^{\infty}\left(\left\{\delta p_{k}^{c}\right\}^{T} \int_{S}\left[t_{k}^{*_{c}}(x)\right]^{T}\{\bar{U}(x)\} d S+\left\{\delta p_{k}^{a}\right\}^{T} \int_{S}\left[t_{k}^{*_{a}}(x)\right]^{T}\{\bar{U}(x)\} d S\right)
$$

Substituting of (7), (8) into variational Eqn. (1), makes it possible to use the standard procedures of the finite element method: integrating for the relevant areas and boundaries of a layer; constructing the local compliance matrices; proceeding to global coordinates; combining the generated matrices and, finally, forming the system of independent algebraic equations for the coefficients of the force vector decomposition at the boundary of each layer:

$$
\left[S_{k}\right]\left\{p_{k}\right\}=\left\{F_{k}\right\}
$$

where $\left[S_{k}\right]$ is the global compliance matrix, which has a band structure, $\left\{F_{k}\right\}$ is the $k$-th harmonic of a given displacement vector along the boundaries of the layers. 
The constructed finite element analog (9) of variational Eqn. (1), owing to the use of nonlinear shape functions (as analytical solution of a stress problem for a single layer) in the FEM (finite element method) semi-analytical scheme, allows us to considerably decrease the number of the unknown. When using this approach, the total number of the unknown in the elastic equilibrium problem for a system of plane-parallel layers is equal to $4 K(N+1)$, where $K$ is the number of terms in a Fourier series retained in expansion (2), and $N$ is the number of layers. Symmetry about the $y$-axis, makes it possible to reduce the number of the unknowns by half.

\section{Test eXAMPLE}

A s a test example, we consider a massless plane, which is under the action of a distributed load. The computational scheme for the problem is presented in Fig. 2.

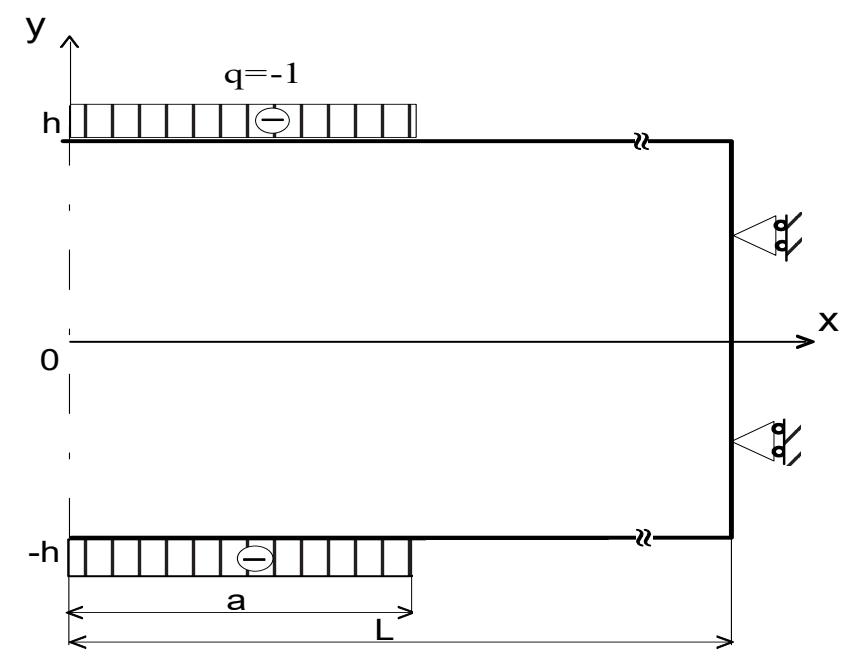

Figure 2: The computational scheme of single layer test system

The specified static boundary conditions are the following:

$$
\begin{array}{llll}
y=+b & \tau_{x y}=0, & \sigma_{y}=\bar{q} ; & (0 \leq x \leq a) \\
y=-b & \tau_{x y}=0, & \sigma_{y}=\bar{q} ; & (0 \leq x \leq a) \\
x=0 ; & x=L & U_{x}=0 . &
\end{array}
$$

An analytical solution of this problem can be constructed with the use of the Fourier series. According to [10], an equation for the Airy stress function [11]:

$$
\frac{\partial^{4} \phi}{\partial x^{4}}+2 \frac{\partial^{4} \phi}{\partial x^{2} \partial y^{2}}+\frac{\partial^{4} \phi}{\partial y^{4}}=0
$$

will be identically satisfied if it is presented as follows:

$$
\phi=\sin \frac{m \pi x}{l} f(y)
$$

where $m$ is any integer number, and function $f(y)$ is a solution of following equation:

$$
\alpha^{4} f(y)-2 \alpha^{2} f^{\prime \prime}(y)+f^{I V}(y)=0
$$


where $\alpha=m \pi / l$.

A general solution of this linear differential equation with constant coefficients takes the following form:

$$
f(y)=C_{1} \operatorname{ch}(\alpha y)+C_{2} \operatorname{sh}(\alpha y)+C_{3} y c h(\alpha y)+C_{4} y s h(\alpha y)
$$

Then a stress function is determined according to the following expression:

$$
\phi=\sin \alpha x\left(C_{1} c h(\alpha y)+C_{2} \operatorname{sh}(\alpha y)+C_{3} y \operatorname{ch}(\alpha y)+C_{4} y s h(\alpha y)\right)
$$

and the relevant stress components are calculated according to the following formulas:

$$
\begin{aligned}
& \sigma_{x}=\frac{\partial^{2} \phi}{\partial y^{2}}=\sin \alpha x\left[C_{1} \alpha^{2} \operatorname{ch}(\alpha y)+C_{2} \alpha^{2} \operatorname{sh}(\alpha y)+C_{3} \alpha(2 \operatorname{sh}(\alpha y)+\alpha y \operatorname{ch}(\alpha y))+C_{4} \alpha(2 \operatorname{ch}(\alpha y)+\alpha y s h(\alpha y))\right] \\
& \sigma_{y}=\frac{\partial^{2} \phi}{\partial x^{2}}=-\alpha^{2} \sin \alpha x\left(C_{1} c h(\alpha y)+C_{2} \operatorname{sh}(\alpha y)+C_{3} y \operatorname{ch}(\alpha y)+C_{4} y \operatorname{sh}(\alpha y)\right) \\
& \tau_{x y}=-\frac{\partial^{2} \phi}{\partial x \partial y}=-\alpha \cos \alpha x\left[C_{1} \alpha \operatorname{sh}(\alpha y)+C_{2} \alpha \operatorname{ch}(\alpha y)+C_{3}(\operatorname{ch}(\alpha y)+\alpha y \operatorname{sh}(\alpha y))+C_{4} \alpha(\operatorname{sh}(\alpha y)+\alpha y \operatorname{ch}(\alpha y))\right]
\end{aligned}
$$

where constants $C_{1}, C_{2}, C_{3}, C_{4}$ are determined by relevant stress boundary conditions.

For the test problem (Fig. 2), the boundary conditions can be represented in the form of symmetric expansion into a Fourier series:

$$
q=A_{0}+\sum_{m=1}^{\infty} A_{m} \cos \frac{m \pi x}{l}
$$

where the expansion coefficients are defined by the following expressions:

$$
A_{0}=\frac{q a}{l}, \quad A_{m}=\frac{1}{l} \int_{-a}^{a} q \cos \frac{m \pi x}{l} d x=\frac{2 q \sin \frac{m \pi a}{l}}{m \pi}
$$

With account of the boundary conditions, the constants $C_{1}, C_{2}, C_{3}, C_{4}$ in expressions for stresses (15):

$$
\begin{aligned}
& C_{1}=-\frac{2 q}{\alpha^{2} \cos \alpha a} \cdot \frac{\operatorname{sh}(\alpha b)+\alpha b c h(\alpha b)}{\operatorname{sh}(2 \alpha b)+2 \alpha b} \\
& C_{4}=\frac{2 q}{\alpha^{2} \cos \alpha a} \cdot \frac{\alpha \operatorname{sh}(\alpha b)}{\operatorname{sh}(2 \alpha b)+2 \alpha c} \\
& C_{2}=C_{3}=0
\end{aligned}
$$

where $q$ - is determined by expression (16).

The results of an analytical solution for middle line $(y=0)$ on which only a normal stress takes place: 


$$
\sigma_{y}=-\frac{q a}{l}-\frac{4 q}{\pi} \sum_{m=1}^{\infty} \frac{\sin \frac{m \pi a}{l}}{m}\left(\frac{\frac{m \pi h}{l} \operatorname{ch}\left(\frac{m \pi h}{l}\right)+\operatorname{sh}\left(\frac{m \pi h}{l}\right)}{\operatorname{sh}\left(\frac{2 m \pi h}{l}\right)+2 \frac{m \pi h}{l}}\right) \cos \frac{m \pi x}{l}
$$

are presented in Tab. 1.

\begin{tabular}{rlll}
\hline $\mathrm{X}, \mathrm{m}$ & $\begin{array}{c}\text { Analytical } \\
\text { solution, } \\
\sigma_{y} / \mathrm{q}\end{array}$ & $\begin{array}{c}\text { Numerical } \\
\text { solution, } \\
\sigma_{y} / \mathrm{q}\end{array}$ & Error,\% \\
\hline 0.0 & -0.69989 & -0.70948 & 1.35169 \\
1.0 & -0.68535 & -0.69497 & 1.38423 \\
2.0 & -0.64334 & -0.65297 & 1.47480 \\
3.0 & -0.57850 & -0.58792 & 1.60227 \\
4.0 & -0.49790 & -0.50668 & 1.73285 \\
5.0 & -0.40988 & -0.41746 & 1.81574 \\
6.0 & -0.32250 & -0.32837 & 1.78762 \\
7.0 & -0.24219 & -0.24603 & 1.56079 \\
8.0 & -0.17298 & -0.17468 & 0.97321 \\
9.0 & -0.11649 & -0.11617 & 0.27546 \\
\hline
\end{tabular}

Table 1: The results of calculation of a vertical stress along the middle line

The calculations were performed for $L=20 m, a=4 m, 2 h=18 m, E=G P a$.

Tab. 1 also presents the results of the numerical solution, which was obtained based on the developed semi-analytical scheme of the finite element method. In the numerical implementation of the finite-dimensional analog, the force vector for the area consisting of three layers and the corresponding right-hand part of Eqn. (9) are given as:

$$
\left\{p_{k}\right\}=\left\{\begin{array}{c}
\sigma_{y y k}(h) \\
\tau_{x y k}(b) \\
\sigma_{y y k}(h / 3) \\
\tau_{x y k}(h / 3) \\
\sigma_{y y k}(-h / 3) \\
\tau_{x y k k}(-h / 3) \\
\sigma_{y y k}(-h) \\
\tau_{x y / k}(-h)
\end{array}\right\}, \quad\left\{F_{k}\right\}=\left\{\begin{array}{c}
q=A_{0}+A_{m} \cos \frac{m \pi x}{l} \\
0 \\
0 \\
0 \\
0 \\
0 \\
q=A_{0}+A_{m} \cos \frac{m \pi x}{l} \\
0
\end{array}\right\}
$$

A comparative analysis of the results of the numerical and analytical solutions shows that the proposed approach for modeling the state of the system of plane-parallel layers ensures fairly accurate calculations.

Thus, the proposed scheme for the assessment of the strain-stress state of a layered rock mass, using the nonlinear shape functions as an analytical solution for a single layer, demonstrates its efficiency in solving the relevant problems with the aid of sufficiently accurate calculations. 
As an example problem, we consider the Upper Kama salt deposit Russia, Perm Krai, which is a layered rock mass mined out by the room-and-pillar method. A computational scheme of the problem with account of symmetry about the $y$ axis is given in Fig. 3. The characteristics of the geological section and the mechanical properties of the examined layers are presented in Tab. 2.

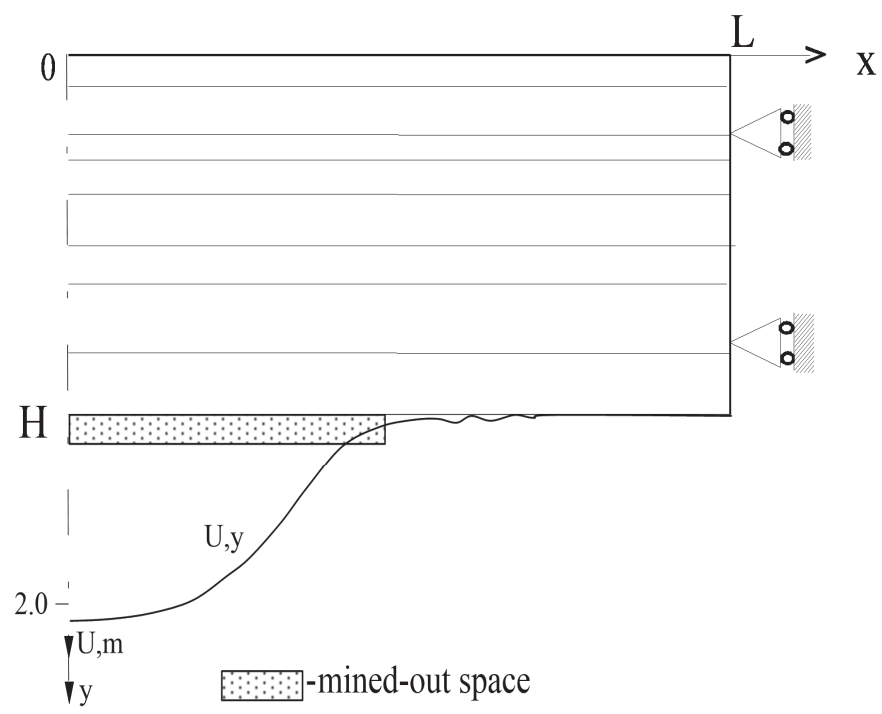

Figure 3: The computational scheme of multilayers system

\begin{tabular}{|c|c|c|c|c|c|}
\hline №. & $\begin{array}{c}\text { Bed, } \\
\text { stratum }\end{array}$ & $\begin{array}{c}\text { Thickness } \\
\text { of layer, } \\
\text { m }\end{array}$ & $\begin{array}{l}\text { Modulus } \\
\text { of } \\
\text { deformation } \\
E, G P a\end{array}$ & $\begin{array}{c}\text { Poisson's } \\
\text { ratio } v\end{array}$ & $\begin{array}{c}\text { Specific } \\
\text { weight } \\
\gamma, \mathrm{KH} / \mathrm{M}^{3}\end{array}$ \\
\hline 1 & Q & 9.1 & 0.10 & 0.30 & 20 \\
\hline 2 & PTs & 18.3 & 0.70 & 0.30 & 23 \\
\hline 3 & TK'T & 50.0 & 1.20 & 0.30 & 24 \\
\hline 4 & SMT & 48.0 & 0.30 & 0.40 & 22 \\
\hline 5 & PP & - & 0.80 & 0.30 & 22 \\
\hline 6 & PKS & 20.0 & 1.20 & 0.30 & 22 \\
\hline 7 & $\mathrm{~K}, \mathrm{I}-\mathrm{K}$ & 5.6 & 0.94 & 0.31 & 21.8 \\
\hline 8 & I, Z-I & 5.7 & 0.91 & 0.31 & 21.7 \\
\hline 9 & Z, ZH-Z & 5.1 & 0.89 & 0.31 & 21.6 \\
\hline 10 & $\begin{array}{c}\mathrm{ZH}, \mathrm{E}- \\
\mathrm{ZH}\end{array}$ & 3.7 & 0.92 & 0.31 & 21.7 \\
\hline 11 & $\mathrm{E}$ & 10.7 & 0.50 & 0.35 & 20 \\
\hline 12 & D-E & 4.0 & 1.0 & 0.30 & 22 \\
\hline 13 & $\mathrm{D}$ & 13.0 & 0.50 & 0.35 & 20 \\
\hline 14 & G-D & 3.0 & 1.0 & 0.30 & 22 \\
\hline 15 & G & 13.0 & 0.50 & 0.35 & 20 \\
\hline 16 & V-G & 5. & 1.0 & 0.30 & 22 \\
\hline 17 & $\mathrm{~V}$ & 10.0 & 0.60 & 0.30 & 22 \\
\hline 18 & B-V & 4.5 & 1.0 & 0.30 & 22 \\
\hline
\end{tabular}

Table 2: Physical and mechanical properties of rocks 
The boundary conditions are specified as follows: the upper boundary is free, at the side boundaries the horizontal displacements are equal to zero, the lower boundary has vertical displacements reflecting the deformation of the top of the upper mined bed as a result of mining operations (Fig. 3.). It is very important that these displacements were obtained as the result of mine geodetic measurements and characterize the real deformation processes in the mined mass.

The initial stress of the undisturbed mass was taken to be lithostatic. In this case $, \sigma_{y}^{0}=\gamma H, \sigma_{x}^{0}=\gamma H v /(1-v)$ where $\gamma$ is the specific weight of rocks, $H$ is the mining depth. Additional stresses were determined by solving the initial problem. The stresses were expanded into a Fourier series, in which 60 harmonics of their symmetric part were retained. The number of the unknowns in the algebraic analog of the problem was 2280.

A specific feature of rocks, containing salt strata, is low values of ultimate tensile strains [12], which at the average is 0.1 $0.2 \%$ and serve as the indicators of the formation of subvertical cracks in the salt beds [13]. The presence of the through tension zone with horizontal strains exceeding the threshold values for saliferous rocks is indicative of the risk of discontinuity in the waterproof stratum and ingress of fresh waters into mine openings.

The distribution of horizontal tensile strains is illustrated in Fig. 4. The obtained results are in good agreement with empirical data on the crack formation in the layers of mined rock strata [13].

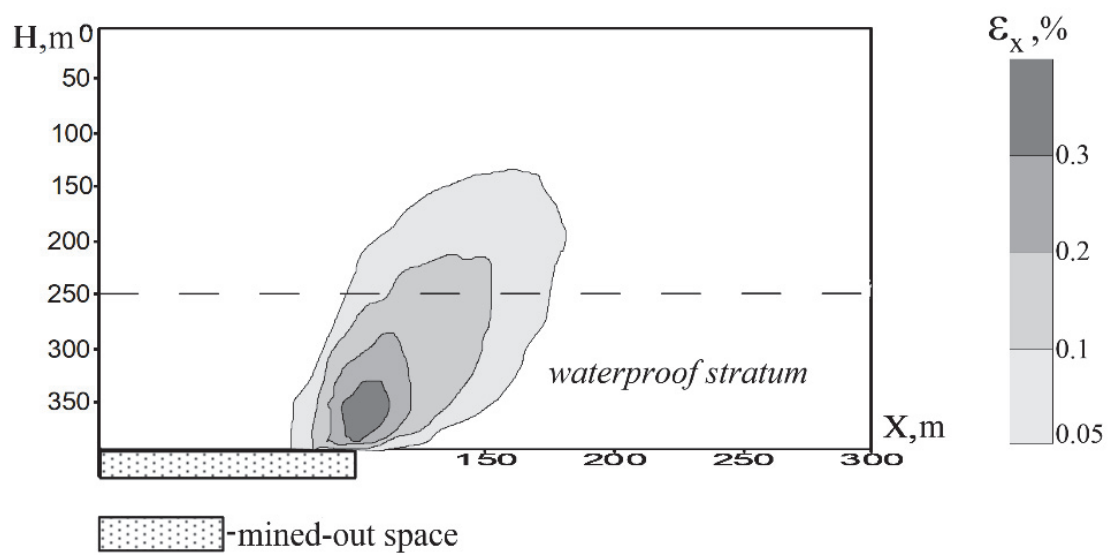

Figure 4: Distribution of horizontal tension strains in the mined-out mass

\section{CONCLUSIONS}

I $\mathrm{n}$ this study, we demonstrated the efficiency of the proposed semi-analytical scheme of the finite element method for assessing the changes in the state of the layered rock mass under the conditions of underground mining based on a rather simple computational algorithm. At the same time, the degree of the impact of mining operations on the rock mass is determined by the boundary conditions and can be generally estimated using the field measurements. Utilization of an actual survey data in geomechanical calculations provides higher validity of waterproof stratum stability estimations and is applied successfully for prompt analysis of mining safety.

\section{ACKNOWLEDGMENTS}

his work has been financed by the Russian Science Foundation (grant No. 19-77-30008).

\section{REFERENCES}

[1] Baryakh, A.A., Asanov, V.A., Samodelkina, N.A., Pankov, I.L., Telegina, E.A. J. (2013). Geomechanical provision of protection of potassium mines from flooding. Gornyi Zhurnal. 6, pp. 30-34. 
[2] Baryakh, A.A., Samodelkina, N.A. J. (2012). Water-tight stratum rupture under large-scale mining. Part II. Journal of Mining Science. 48(6), pp. 954-961.

[3] Maier, G., Novatti, G. J. (1987). Boundary element elastic analysis of layered soils by successive stiffness or compliance methods, Int. J. Numerical and Analytical Meth. in Geomechanics, 11(5), pp. 435-447.

[4] Filippov, N.A. J. (1979). On calculating the strain-stress state of layered rock mass. Physical and technical problems of mineral resource development, 2, pp. 3-10.

[5] Filippov, N.A. (1982). The Fourier method in problems of mechanics of layered media under non-traditional contact conditions. In: Problems in mechanics of solid deformable bodies, 14, pp. 221-229.

[6] Linkov, A.M., Filippov, N.A., Fot, K.K. (1989). On the solution of problems for layered media by means of the Fourier series expansion. In: "Studies of mechanics of building constructions and materials", LISI.

[7] Linkov, A.M., Filippov, N.A., Fot, K.K. (1988). Difference equations in layered media problems. VINITI, Leningrad.

[8] Vasidzu K. (1987). Variational methods in the theory of elasticity and plasticity. M.: Mir.

[9] Petrishin, V.N., Privarnikov, A.K. (1965). Main boundary problems of the theory of elasticity for multilayered bases. In: Applied mechanics, 4(1), pp. 58-66.

[10] Ribiere, M.C. (1898). Compt. Rend. 126, pp. 402-404, 1190-1192.

[11] Timoshenko, S.P., Goodier, J. (1979). Theory of elasticity: Translated from English / Edited by Shapiro G.S. M.: Nauka. Main office of physical and mathematical literature.

[12] Baryakh, A.A., Asanov, V.A., Pankov, I.L. (2008). Physical and mechanical properties of saliferous rocks of the Upper Kama potash deposit. Perm.

[13] Baryakh, A.A. J. (1995). Geomechanical aspects of protection of potassium mines against flooding. Izvestiya vysshikh uchebnykh zavedenii. Gornyi Zhurnal, 6. pp.185-186. 